\title{
Link between the Relativistic Canonical Quantum Mechanics and the Dirac Equation
}

\author{
V.M. Simulik*, I.Yu. Krivsky \\ Institute of Electron Physics, National Academy of Sciences, Uzhgorod, 88000, Ukraine \\ *Corresponding Author: vsimulik@gmail.com
}

Copyright (C2014 Horizon Research Publishing All rights reserved.

\begin{abstract}
The foundations of the relativistic canonical quantum mechanics of a fermionic doublet on the basis of the Schrödinger-Foldy equation of motion are formulated. A brief review of the different ways of the Dirac equation derivation is given. The Dirac equation is derived from the Schrödinger-Foldy equation of the relativistic canonical quantum mechanics. It is shown that our model does not need applying of the negative mass concept of the antiparticle.
\end{abstract}

Keywords Relativistic Canonical Quantum Mechanics, the Schrödinger-Foldy Equation, the Dirac Equation, the Spinor Field

\section{Introduction}

A deep analysis of the problems of relativistic quantum mechanics is given. We investigate here the relativistic canonical quantum mechanics (RCQM) and its link with the Dirac equation. Unfortunately, there still exist scientists of "old school", who identify the relativistic quantum mechanics and the Dirac equation. Indeed, the old concept that the Dirac equation gives the relativistic quantum-mechanical description of the fermionic doublet is still widespread.

Nevertheless, in some principal quantum-mechanical problems the Dirac's description of the fermionic doublet is not satisfactory. First of all, it is related to the consistent quantum-mechanical interpretation. L. Foldy and S. Wouthuysen [1] suggested a nonlocal canonical formulation of the equation for the spinor field. The canonical representation for the spinor field was introduced. In this representation [1-3], first of all for the electron having spin $\vec{s}=\frac{1}{2} \vec{\sigma}$, many quantum-mechanical details of the Dirac model were clarified. The equation $i \partial_{t} f(x)=$ $\sqrt{m^{2}-\Delta} f(x), f=\left|\begin{array}{l}f^{1} \\ f^{2}\end{array}\right|$, was used. Such description [1-3] is adequate in the sense of its comparison with the nonrelativistic Schrödinger model of the electron. The direct relativistic analogue of the Schrödinger equation is the spinless Salpeter equation [4-6] for the onecomponent wave function: $i \partial_{t} f(x)=\sqrt{m^{2}-\Delta} f(x)$.

The relativistic equations mentioned above can in an obvious way be generalized for particle multiplets with arbitrary spin. For the case of arbitrary spin and multicomponent wave functions, we suggested [7, 8] to call such type of equations as the Schrödinger-Foldy equations. The important contribution of L. Foldy [1-3] into the consideration and analysis of the spinor field in the nonlocal canonical representation (and his analysis of the principles of heredity and correspondence with nonrelativistic quantum mechanics) was taken into account.

The analysis of the 4-component spinor field [1-3] enabled the authors of these papers to discern the quantum-mechanical interpretation of the Dirac equation. For these purposes, the Dirac equation was transformed into another representation $i \partial_{t} f(x)=$ $\gamma^{0} \sqrt{m^{2}-\Delta} f(x)$, which is today called the FoldyWouthuysen (FW) representation. Modern, coherent and detailed consideration of the Dirac theory is presented in the B. Thaller's monograph [9].

At first, we consider briefly the 2-component case of the Schrödinger-Foldy equation for the electron. Then we proceed to the Schrödinger-Foldy equation for the 4-component wave function of spin 1/2 doublet (in all essential details). In this important example the axiomatic formulation of corresponding relativistic canonical quantum mechanics (RCQM) is presented briefly. The derivation of the Dirac equation directly from the Schrödinger-Foldy equation (without any additional assumptions) is given.

We started the investigation of this subject in Ref. [10]. Here the basic principles of the RCQM for the spin $s=\frac{1}{2}$ doublet and the derivation of the Dirac equation from this model are under further consideration. The foundations of RCQM were given in $[1-4,7-8,10]$. Here the mathematically well-defined description on the level of modern axiomatic approaches to the field theory [11] is provided.

\section{Motivation and goals}

The significance of the Dirac equation and its widerange application in different models of theoretical physics (QED, QHD, theoretical atomic and nuclear physics, solid systems) is well-known. Even the first analysis of this equation enabled Dirac to give a theoretical prediction of the positron, which was discovered 
experimentally by Anderson in 1932. The recent wellknown application of the massless Dirac equation to the graphene ribbons is an example of possibilities of this equation. In our recent publications [12-16] we were able to extend the domain for the application of the Dirac equation. We proved [12-16] that this equation has not only fermionic but also bosonic features, can describe not only fermionic, but also the bosonic states.

The deep analysis of the Dirac equation is also the subject of recent studies by other authors [17-20]. The authors of [17] use the theory of fractional powers of linear operators to construct a general (analytic) representation theory for the square-root energy operator $H=\gamma^{0} \sqrt{m^{2}-\Delta}$ of relativistic quantum theory, which is valid for all values of the spin. An analytical diagonalization of the full (minimal coupling) Dirac equation into particle and antiparticle components was constructed in Ref. [18]. The diagonalization is analytical in the sense that it is achieved without transforming the wavefunctions, as is done by the FW method, and reveals the nonlocal time behavior of the particle-antiparticle relationship. In Ref. [19] different spin operators for the Dirac equation proposed in the literature are reviewed. The authors select the spin operator, which is equivalent to the Newton-Wigner spin operator and the FW mean-spin operator. It is shown [20] that the covariant relativistic spin operator is equivalent to the spin operator commuting with the free Dirac Hamiltonian. We mention above only the papers somehow related to our study. The list of all contemporary authors dealing with the Dirac equation is very long.

Therefore, the new ways of the derivation of the Dirac equation are important. These new ways automatically visualize the ground principles, which are in the foundations of the description of the elementary particles on the basis of this equation. Hence, the active consideration of the different ways of the Dirac equation derivation is the subject of many contemporary publications, where they originates from the different basic principles and assumptions.

In Section 8 a brief review of the different methods of the Dirac equation derivation is given.

Today we present a new derivation of the Dirac equation (Section 9). We derive the Dirac equation from the 4-component Schrödinger-Foldy equation of the RCQM. We postulate the Schrödinger-Foldy equation (Section 4) and construct the corresponding formalism of RCQM as the most fundamental model of fermionic doublet (sections 5,6). At first, the brief axiomatic formulation of the RCQM foundations is given (section 6). After that, the operator, which transforms the SchrödingerFoldy equation into the Dirac equation, is written down (section 9). Therefore, a new way of the Dirac equation derivation is presented.

Our main goal is to answer the question "Does there exist a more fundamental model of a "particle doublet" (as an elementary fundamental object), from which the Dirac equation (and its content) would follow directly and unambiguously?" We are able to demonstrate that axiomatically formulated RCQM of a particleantiparticle doublet of spin $s=\frac{1}{2}$ should be chosen as such a model. In the text below the illustration of this assertion is given for the example of electron-positron doublet, $e^{-} e^{+}$-doublet

\section{$3 \quad$ Notations and main definitions}

The model of RCQM for the elementary particle with $m>0$ and spin $s=\frac{1}{2}$, which satisfy the SchrödingerFoldy equation $i \partial_{t} f(x)=\sqrt{m^{2}-\Delta} f(x) ; x \in$ $\mathrm{M}(1,3), f=\left|\begin{array}{l}f^{1} \\ f^{2}\end{array}\right|, \int d^{3} x|\varphi(x)|^{2}<\infty$, was suggested and approved in [1-3]. This model can be easily generalized to the case of arbitrary $\vec{s}$-multiplet, i. e. the "elementary object" with mass $m$ and spin $\vec{s} \equiv\left(s^{j}\right)=$ $\left(s_{23}, s_{31}, s_{12}\right):\left[s^{j}, s^{l}\right]=i \varepsilon^{j l n} s^{n}$, where $\varepsilon^{j l n}$ is the LeviCivita tensor and $s^{j}=\varepsilon^{j \ell n} s_{\ell n}$ are the Hermitian $\mathrm{M} \times \mathrm{M}$ matrices - the generators of M-dimensional representation of the spin group $\mathrm{SU}(2)$ (universal covering of the $\mathrm{SO}(3) \subset \mathrm{SO}(1,3)$ group).

In this article, we present the detailization of such generalization at the example of the spin $s=\frac{1}{2}$ fermionic doublet. All mathematical and physical details of consideration, related to the choice of the specific form of the spin $\vec{s}$ doublet, at the example of $e^{-} e^{+}$-doublet are illustrated.

We choose here the standard relativistic concepts, definitions and notations in the form convenient for our consideration. For example, in the Minkowski space-time

$$
\begin{gathered}
\mathrm{M}(1,3)=\left\{x \equiv\left(x^{\mu}\right)=\left(x^{0}=t, \vec{x} \equiv\left(x^{j}\right)\right)\right\} ; \\
\mu=\overline{0,3}, j=1,2,3,
\end{gathered}
$$

$x^{\mu}$ are Cartesian (covariant) coordinates of the points of the physical space-time in the arbitrary-fixed inertial reference frame (IRF). We use the system of units with $\hbar=c=1$. The metric tensor is given by

$$
\begin{gathered}
g^{\mu \nu}=g_{\mu \nu}=g_{\nu}^{\mu},\left(g_{\nu}^{\mu}\right)=\operatorname{diag}(1,-1,-1,-1) ; \\
x_{\mu}=g_{\mu \nu} x^{\mu},
\end{gathered}
$$

summation over twice repeated indices is implied.

The analysis of the relativistic invariance of an arbitrary physical model requires, as a first step, consideration of its invariance with respect to the proper ortochronous Lorentz $\mathrm{L}_{+}^{\uparrow}=\operatorname{SO}(1,3)=\left\{\Lambda=\left(\Lambda_{\nu}^{\mu}\right)\right\}$ and Poincaré $\left.\mathrm{P}_{+}^{\uparrow}=\mathrm{T}(4) \times\right) \mathrm{L}_{+}^{\uparrow} \supset \mathrm{L}_{+}^{\uparrow}$ groups. This invariance in an arbitrary relativistic model is the implementation of the Einstein's relativity principle in the form of special relativity. Note that the mathematical correctness requires one to consider the invariance mentioned above as the invariance with respect to the universal coverings $\mathcal{L}=\mathrm{SL}(2, \mathrm{C})$ and $\mathcal{P} \supset \mathcal{L}$ of the groups $\mathrm{L}_{+}^{\uparrow}$ and $\mathrm{P}_{+}^{\uparrow}$, respectively.

For the group $\mathcal{P}$ we choose real parameters $a=$ $\left(a^{\mu}\right) \in \mathrm{M}(1,3)$ and $\varpi \equiv\left(\varpi^{\mu \nu}=-\varpi^{\nu \mu}\right)$, whose physical meaning is well-known. For the standard $\mathcal{P}$ generators $\left(p_{\mu}, j_{\mu \nu}\right)$ we use commutation relations in the manifestly covariant form

$$
\begin{gathered}
{\left[p_{\mu}, p_{\nu}\right]=0,\left[p_{\mu}, j_{\rho \sigma}\right]=i g_{\mu \rho} p_{\sigma}-i g_{\mu \sigma} p_{\rho}} \\
{\left[j_{\mu \nu}, j_{\rho \sigma}\right]=-i\left(g_{\mu \rho} j_{\nu \sigma}+g_{\rho \nu} j_{\sigma \mu}+g_{\nu \sigma} j_{\mu \rho}+g_{\sigma \mu} j_{\rho \nu}\right)}
\end{gathered}
$$




\section{The equation of motion of rela-} tivistic canonical quantum mechanics

In this section we consider the comparison of Schrödinger-Foldy and FW equations for the fermionic doublet. On this bases we demonstrate why the Schrödinger-Foldy equation should be chosen as the main equation of motion in RCQM.

The Schrödinger-Foldy equation for the fermionic spin $1 / 2$ doublet is given by

$$
i \partial_{t} f(x)=\sqrt{m^{2}-\Delta} f(x)
$$

where

$$
f \equiv \operatorname{column}\left(f^{1}, f^{2}, f^{3}, f^{4}\right) .
$$

This equation, similarly to the nonrelativistic 4component Schrödinger equation

$$
i \partial_{t} f(x)=\frac{\vec{p}^{2}}{2 m} f(x)
$$

(involving also the internal degrees of freedom, spin etc.) is considered in the quantum-mechanical Hilbert space

$$
\begin{gathered}
\mathrm{H}^{3,4}=\mathrm{L}_{2}\left(\mathrm{R}^{3}\right) \otimes \mathrm{C}^{\otimes 4}=\left\{f=\left(f^{\alpha}\right): \mathrm{R}^{3} \rightarrow \mathrm{C}^{\otimes 4} ;\right. \\
\left.\int d^{3} x|f(t, \vec{x})|^{2}<\infty\right\},
\end{gathered}
$$

(strictly speaking, the rigged Hilbert space is necessary; this space is introduced in (14) and is explained in the paragraphs after this equation) where $d^{3} x$ is the Lebesgue measure in the space $\mathrm{R}^{3} \subset \mathrm{M}(1,3)$ of the eigenvalues of the position operator $\vec{x}$ of the Cartesian coordinate of the doublet in an arbitrary-fixed IRF. In $(4)-(7)$ and below the two upper components $f^{1}, f^{2}$ of the vector $f \in \mathrm{H}^{3,4}$ are the components of the electron wave function $\varphi_{-}$and the two lower components $f^{3}, f^{4}$ are those of the positron wave function $\varphi_{+}$.

The general solution of the Schrödinger-Foldy equation (4), similarly to the general solution of the nonrelativistic 4-component Schrödinger equation (6), is given by

$$
\begin{gathered}
f(x)=\left|\begin{array}{c}
f_{e^{-}} \\
f_{e^{+}}
\end{array}\right|=\frac{1}{(2 \pi)^{\frac{3}{2}}} \int d^{3} k e^{-i k x} \\
{\left[a_{+}^{-}(\vec{k}) \mathrm{d}_{1}+a_{-}^{-}(\vec{k}) \mathrm{d}_{2}+a_{-}^{+}(\vec{k}) \mathrm{d}_{3}+a_{+}^{+}(\vec{k}) \mathrm{d}_{4}\right],}
\end{gathered}
$$

where

$$
k x \equiv \omega t-\vec{k} \vec{x}, \quad \omega \equiv \sqrt{\vec{k}^{2}+m^{2}},
$$

the 4-columns $\mathrm{d}_{\alpha}$ are the Cartesian orts in the space $\mathrm{C}^{\otimes 4} \subset \mathrm{H}^{3,4}$

$$
\mathrm{d}_{1}=\left|\begin{array}{c}
1 \\
0 \\
0 \\
0
\end{array}\right|, \mathrm{d}_{2}=\left|\begin{array}{c}
0 \\
1 \\
0 \\
0
\end{array}\right|, \mathrm{d}_{3}=\left|\begin{array}{c}
0 \\
0 \\
1 \\
0
\end{array}\right|, \mathrm{d}_{4}=\left|\begin{array}{c}
0 \\
0 \\
0 \\
1
\end{array}\right|
$$

the functions $a_{+}^{-}(\vec{k}), a_{-}^{-}(\vec{k})$ are the quantummechanical momentum-spin amplitudes of the particle with charge -e and eigen values of spin projection $+1 / 2$ and $-1 / 2 ; a_{-}^{+}(\vec{k}), a_{+}^{+}(\vec{k})$ are the quantum-mechanical momentum-spin amplitudes of the antiparticle with charge $+\mathrm{e}$ and eigen values of spin projection $-1 / 2$ and $+1 / 2$, respectively.

In the general solution (8) we use the modern experimentally verified understanding of the positron as the "mirror mapping" of the electron. Such understanding leads to the specific postulation of the explicit forms of the charge sign and spin operators (see equation (24) in [7]), which determine the form of the solution (8).

Contrary to the Schrödinger-Foldy equation (4), the FW equation has the form

$$
\begin{gathered}
i \partial_{t} \phi(x)=\gamma^{0} \sqrt{m^{2}-\Delta} \phi(x), \\
\gamma^{0}=\left|\begin{array}{ll}
\mathrm{I}_{2} & 0 \\
0 & -\mathrm{I}_{2}
\end{array}\right|, \quad \mathrm{I}_{2}=\left|\begin{array}{ll}
1 & 0 \\
0 & 1
\end{array}\right|,
\end{gathered}
$$

and its general solution is given by

$$
\begin{gathered}
\phi(x)=\frac{1}{(2 \pi)^{\frac{3}{2}}} \int d^{3} k\left\{e^{-i k x}\left[a_{+}^{-}(\vec{k}) \mathrm{d}_{1}+a_{-}^{-}(\vec{k}) \mathrm{d}_{2}\right]+\right. \\
\left.e^{i k x}\left[a_{-}^{*+}(\vec{k}) \mathrm{d}_{3}+a_{+}^{*+}(\vec{k}) \mathrm{d}_{4}\right]\right\}
\end{gathered}
$$

It means that $\phi(x)=\left|\begin{array}{c}f_{e^{-}} \\ f_{e^{+}}^{*}\end{array}\right|$.

The equations (4) and (11) are different due to the presence of $\gamma^{0}$ in (11). Owing to this fact the general solutions (8) and (12) are also different. The solution (8) is the direct sum of the electron $f_{e^{-}}$and the positron $f_{e^{+}}$ quantum-mechanical wave functions. The solution (12) is the direct sum of the electron $f_{e^{-}}$and the complex conjugated positron $f_{e^{+}}^{*}$ wave functions. Furthermore, the solution (8) contains only positive energy states both for the electron and positron, whereas the solution (12) contains the positive energy states of the electron and negative energetic states of the positron.

Note that solution (8) for the wave function of spin $1 / 2$ doublet is expressed in the terms of relativistic de Broglie waves for the electron and the positron

$$
\varphi_{\vec{k} \mathrm{~A}}(t, \vec{x})=\frac{1}{(2 \pi)^{\frac{3}{2}}} e^{-i \omega t+i \vec{k} \vec{x}} \mathrm{~d}_{\mathrm{A}}, \quad \mathrm{A}=1,2,3,4 .
$$

The expressions (13) are the fundamental (basis) solutions of the equation (4), they do not belong to the Hilbert space (7) (their $\mathrm{H}^{3,4}$-norms are equal to the infinity). The mathematical correctness of the consideration is ensured by applying the rigged Hilbert space

$$
\mathrm{S}^{3,4} \equiv \mathrm{S}\left(\mathrm{R}^{3}\right) \times \mathrm{C}^{4} \subset \mathrm{H}^{3,4} \subset \mathrm{S}^{3,4 *} .
$$

Here $\mathrm{S}^{3,4}$ is the 4-component Schwartz test function space over the space $\mathrm{R}^{3} \subset \mathrm{M}(1,3)$, and $\mathrm{S}^{3,4 *}$ is the space of 4-component Schwartz generalized functions, which is conjugated to the Schwartz test function space $\mathrm{S}^{3,4}$ by the corresponding topology (see, e. g., [21]). Strictly speaking, the mathematical correctness of consideration demands one to make the calculations in the space $\mathrm{S}^{3,4 *}$ 
of generalized functions, i. e. with the application of cumbersome functional analysis.

Nevertheless, we take into account that the Schwartz test function space $\mathrm{S}^{3,4}$ in the triple $(22)$ is kernel. It means that $\mathrm{S}^{3,4}$ is dense both in quantum-mechanical space $\mathrm{H}^{3,4}$ and in the space of generalized functions $\mathrm{S}^{3,4 *}$. Therefore, any physical state $f \in \mathrm{H}^{3,4}$ can be approximated with an arbitrary accuracy by the corresponding elements of the Cauchy sequence in $\mathrm{S}^{3,4}$, which converges to the given $f \in \mathrm{H}^{3,4}$ or to the given $f \in \mathrm{S}^{3,4 *}$. Further, taking into account the requirement to measure the arbitrary value of the model with non-absolute accuracy, it means that all specific calculations can be performed within the Schwartz test function space $\mathrm{S}^{3,4}$.

Note that if the general solution $f(x)$ (8) belongs to the $\mathrm{S}^{3,4}$ then the amplitudes $a_{+}^{-}(\vec{k}), a_{-}^{-}(\vec{k}), a_{-}^{+}(\vec{k}), a_{+}^{+}(\vec{k})$ also belong to the test function space $\mathrm{S}\left(\mathrm{R}_{\vec{k}}^{3}\right)$. It is sufficient for the approximation of any experimental situation.

Contrary to this situation, even if amplitudes $a$ in (12) belong to the $\mathrm{S}\left(\mathrm{R}_{\vec{k}}^{3}\right)$, the general solution (12) of the FW equation (11) does not belong to the quantummechanical Hilbert space (7) (due to the indefinite metric in the space of solutions (12)). Nevertheless, the mathematical correctness of consideration is ensured in the space $S^{3,4} \subset S^{3,4 *}$ due to the fact that $S^{3,4}$ is dense in $\mathrm{S}^{3,4 *}$.

Therefore, we are able to demonstrate the difference between the Schrödinger-Foldy equation (4) and the FW equation (11) in the quantum-mechanical description of the fermionic doublet. Despite the fact that in the FW representation one can discern some quantummechanical aspects, however, in general the FW equation does not guarantee a detailed quantum-mechanical description of the fermionic doublet (as well as the Dirac equation).

Hence, we postulate the Schrödinger-Foldy equation (4) to be the RCQM equation of motion for the fermionic spin $1 / 2$ doublet and construct the corresponding canonical quantum-mechanical formalism.

\section{Relativistic canonical quantum mechanics of the single spin $1 / 2$ fermion}

Before considering the spin $1 / 2$ particle doublet it is useful to consider briefly the RCQM of the single spin $1 / 2$ fermion.

The Schrödinger-Foldy equation is given by

$$
i \partial_{t} f(x)=\sqrt{m^{2}-\Delta} f(x), \quad f=\left|\begin{array}{l}
f^{1} \\
f^{2}
\end{array}\right| .
$$

The space of states is the following

$$
\mathrm{S}^{3,2} \subset \mathrm{H}^{3,2} \subset \mathrm{S}^{3,2 *} .
$$

The generators of SU(2)-spin have the explicit form

$$
\vec{s}=\frac{1}{2} \vec{\sigma}, \quad\left[s^{j}, s^{\ell}\right]=i \varepsilon^{j \ell n} s^{n},
$$

where $\vec{\sigma}$ are the standard Pauli matrices

$$
\sigma^{1}=\left|\begin{array}{ll}
0 & 1 \\
1 & 0
\end{array}\right|, \quad \sigma^{2}=\left|\begin{array}{ll}
0 & -i \\
i & 0
\end{array}\right|, \quad \sigma^{3}=\left|\begin{array}{ll}
1 & 0 \\
0 & -1
\end{array}\right|
$$

The Casimir operator is given by

$$
\vec{s}^{2}=\frac{3}{4} \mathrm{I}_{2}=\frac{1}{2}\left(\frac{1}{2}+1\right) \mathrm{I}_{2}, \quad \mathrm{I}_{2}=\left|\begin{array}{ll}
1 & 0 \\
0 & 1
\end{array}\right| .
$$

The general solution of the Schrödinger-Foldy equation (15) is given by

$$
f(x)=\frac{1}{(2 \pi)^{\frac{3}{2}}} \int d^{3} k e^{-i k x}\left[a_{+}^{-}(\vec{k}) \mathrm{d}_{1}+a_{-}^{-}(\vec{k}) \mathrm{d}_{2}\right],
$$

where the notations (9) are used. The orts of 2dimensional Cartesian basis have the form

$$
\mathrm{d}_{1}=\left|\begin{array}{l}
1 \\
0
\end{array}\right|, \mathrm{d}_{2}=\left|\begin{array}{l}
0 \\
1
\end{array}\right|
$$

The solution (20) is associated with the stationary complete set of operators $\vec{p}, s^{3}=s_{z}, g=-e$ of the momentum, spin projection and sign of the charge of the spin $1 / 2$ fermion, respectively.

The equations on eigen values of the spin projection operator $s^{3}=\frac{1}{2}\left|\begin{array}{ll}1 & 0 \\ 0 & -1\end{array}\right|$ are given by

$$
s^{3} \mathrm{~d}_{1}=\frac{1}{2} \mathrm{~d}_{1}, \quad s^{3} \mathrm{~d}_{2}=-\frac{1}{2} \mathrm{~d}_{2} .
$$

The interpretation of the amplitudes $a_{+}^{-}(\vec{k}), a_{-}^{-}(\vec{k})$ follows from the equations (22) and similar equations on the eigen values of operators $\vec{p}, g=-e$ from stationary complete set of operators. The functions $a_{+}^{-}(\vec{k}), a_{-}^{-}(\vec{k})$ are the quantum-mechanical momentum-spin amplitudes of the fermion with charge -e and eigen values of spin projection $+1 / 2$ and $-1 / 2$, respectively.

The Schrödinger-Foldy equation (15) is invariant with respect to the unitary representation

$$
(a, \varpi) \rightarrow U(a, \varpi)=\exp \left(-i a^{0} \widehat{\omega}-i \vec{a} \widehat{\vec{p}}-\frac{i}{2} \varpi^{\mu \nu} \widehat{j}_{\mu \nu}\right)
$$

of the Poincaré group $\mathcal{P}$. The corresponding generators are given by

$$
\begin{gathered}
\widehat{p}_{0}=\widehat{\omega} \equiv \sqrt{-\Delta+m^{2}}, \quad \widehat{p}_{\ell}=i \partial_{\ell}, \\
\widehat{j}_{\ell n}=x_{\ell} \widehat{p}_{n}-x_{n} \widehat{p}_{\ell}+s_{l n} \equiv \widehat{m}_{\ell n}+s_{\ell n},
\end{gathered}
$$

$$
\widehat{j}_{0 \ell}=-\widehat{j}_{\ell 0}=t \widehat{p}_{\ell}-\frac{1}{2}\left\{x_{\ell}, \widehat{\omega}\right\}-\left(\frac{s_{\ell n} \widehat{p}_{n}}{\widehat{\omega}+m} \equiv \breve{s}_{\ell}\right),
$$

where the spin $1 / 2 \mathrm{SU}(2)$ generators $\vec{s}=\left(s^{\ell n}\right)$ are given in (17).

The validity of this assertion is verified by the following three steps. (i) The calculation that the $\mathcal{P}$ generators $(24),(25)$ commute with the operator $i \partial_{0}-\widehat{\omega}$ of the Schrödinger-Foldy equation (15). (ii) The verification that the $\mathcal{P}$-generators (24), (25) satisfy the commutation relations (3) of the Lie algebra of the Poincaré 
group $\mathcal{P}$. (iii) The proof that generators $(24)$ realize the spin $1 / 2$ representation of this group. Therefore, the Bargman-Wigner classification on the basis of the corresponding Casimir operators calculation should be given.

These three steps can be fulfilled by direct and noncumbersome calculations. The corresponding Casimir operators have the form

$$
\begin{gathered}
p^{2}=\widehat{p}^{\mu} \widehat{p}_{\mu}=m^{2}, \\
W=w^{\mu} w_{\mu}=m^{2} \vec{s}^{2}=\frac{1}{2}\left(\frac{1}{2}+1\right) m^{2} \mathrm{I}_{2},
\end{gathered}
$$

where $\mathrm{I}_{2}$ is given in (19).

The brief scheme of the formulation of the RCQM foundations, which is given above for the spin $1 / 2$ fermion, demonstrates the possibility of such scheme to be presented in the form of corresponding axioms. In next section for the example of spin $1 / 2$ particle doublet, which is important for the goals of our considerations, we give the axiomatic formulation of the RCQM foundations.

\section{Relativistic canonical quantum mechanics of the spin $1 / 2$ Fermi-doublet}

The axioms of the model are formulated on the level of correctness of von Neuman's monograph [22]. Requirements of such physically verified principles as the principle of relativity with respect to the tools of cognition (PRTC), principle of heredity $(\mathrm{PH})$ with both classical mechanics of single mass point and nonrelativistic quantum mechanics (and the principle of correspondence $(P C)$ with these theories), and also the Einstein principle of relativity (EPR), are taken into consideration. The last principle requires first of all the special relativity $(S R)$ to be taken into account.

The basic axioms of the model (we present here the brief consideration) as the mathematical assertions have the form of the following statements.

On the space of states. The space of states of isolated $e^{-} e^{+}$-doublet in an arbitrarily-fixed inertial frame of reference (IFR) in its $\vec{x}$-realization is the Hilbert space $\mathrm{H}^{3,4}$ (7) of complex-valued 4-component squareintegrable functions of $x \in \mathrm{R}^{3} \subset \mathrm{M}(1,3)$ (similarly, in momentum, $\vec{p}$-realization). Here $\vec{x}$ and $\vec{p}$ are the operators of canonically conjugated dynamical variables of $e^{-} e^{+}$-doublet, and the vectors $f, \tilde{f}$ in $\vec{x}$ - and $\vec{p}$-realizations are linked by the 3 -dimensional Fourier transformation (the variable $t$ is the parameter of timeevolution).

The mathematical correctness of the consideration demands the application of the rigged Hilbert space (14), where the Schwartz test function space $S^{3,4}$, which is the verified tool of the PRTC implementation, is the kernel (i. e., it is dense both in $\mathrm{H}^{3,4}$ and in the space $\mathrm{S}^{3,4 *}$ of the generalized Schwartz functions). Such application allows us to perform, without any loss of generality, all necessary calculations in the space $S^{3,4}$ on the level of correct differential and integral calculus. The more detailed consideration is given in paragraphs after the definition (14).

On the time evolution of the state vectors. The time dependence of the state vectors $f \in \mathrm{H}^{3,4}$ (time $\mathrm{t}$ is the parameter of evolution) is given either in the integral form by the unitary operator

$$
u\left(t_{0}, t\right)=\exp \left[-i \widehat{\omega}\left(t-t_{0}\right)\right] ; \quad \widehat{\omega} \equiv \sqrt{-\Delta+m^{2}},
$$

(below $t_{0}=t$ is put), or in the differential form by the Schrödinger-Foldy equation of motion (4). Here the operator $\widehat{\omega} \equiv \sqrt{-\Delta+m^{2}}$ is the relativistic analog of the energy operator (Hamiltonian) of nonrelativistic quantum mechanics. The Minkowski space-time $\mathrm{M}(1,3)$ is pseudo Euclidean with metric $g=\operatorname{diag}(+1,-1,-1,-1)$.

The general solution of the Schrödinger-Foldy equation of motion (4) is given by (8).

On the fundamental dynamical variables. The dynamical variable $\vec{x} \in \mathrm{R}^{3} \subset \mathrm{M}(1,3)$ (as well as the variable $\vec{k} \in \mathrm{R}_{\vec{k}}^{3}$ ) represents the external degrees of freedom of the $e^{-} e^{+}$-doublet. The spin $\vec{s}$ of the $e^{-} e^{+}$-doublet is the first in the list of the carriers of the internal degrees of freedom. Taking into account the Pauli principle and the fact that experimentally positron is observed as the mirror reflection of an electron, the operators of the charge sign and the spin of $e^{-} e^{+}$-doublet are taken in the form

$$
g \equiv-\gamma^{0}=\left|\begin{array}{ll}
-\mathrm{I}_{2} & 0 \\
0 & \mathrm{I}_{2}
\end{array}\right|, \quad \vec{s}=\frac{1}{2}\left|\begin{array}{cc}
\vec{\sigma} & 0 \\
0-C \vec{\sigma} C
\end{array}\right|,
$$

where $\vec{\sigma}$ are the standard Pauli matrices (18), $C$ is the operator of complex conjugation, $\mathrm{I}_{2}$ is explained in (19). The spin matrices (29) satisfy the commutation relations of the algebra of $\mathrm{SU}(2)$ group

$\vec{s} \equiv\left(s^{j}\right)=\left(s_{23}, s_{31}, s_{12}\right):\left[s^{j}, s^{l}\right]=i \varepsilon^{j l n} s^{n} ; \varepsilon^{123}=+1$.

where $\varepsilon^{j l n}$ is the Levi-Civita tensor and $s^{j}=\varepsilon^{j \ell n} s_{\ell n}$ are the Hermitian $4 \times 4$ matrices - the generators of a 4-dimensional reducible representation of the spin group $\mathrm{SU}(2)$ (universal covering of the $\mathrm{SO}(3) \subset \mathrm{SO}(1,3)$ group).

On the algebra of observables. Using the operators of canonically conjugated coordinate $\vec{x}$ and momentum $\vec{p}$ (where $\left[x^{j}, p^{\ell}\right]=i \delta_{j \ell}$ in $\mathrm{H}^{3,4}$ ), being completed by the operators $\vec{s}$ and $g$, we construct the algebra of observables (according to the $\mathrm{PH}$ ) as the Hermitian functions of $10\left(\vec{x}, \vec{p} \vec{s},-\gamma^{0}\right)$ generating elements of the algebra.

On the relativistic invariance of the theory. This invariance (implementation of the SR) is ensured by the proof of the invariance of the Schrödinger-Foldy equation (4) with respect to the unitary representation of the universal covering $\mathcal{P} \supset \mathcal{L}=\mathrm{SL}(2, \mathrm{C})$ of the proper ortochronous Poincaré group $\left.\mathrm{P}_{+}^{\uparrow}=\mathrm{T}(4) \times\right) \mathrm{L}_{+}^{\uparrow} \supset \mathrm{L}_{+}^{\uparrow}$. Here $\mathcal{L}=\operatorname{SL}(2, \mathrm{C})$ is the universal covering of proper ortochronous Lorentz group $\mathrm{L}_{+}^{\uparrow}$.

The generators of the fermionic $\mathcal{P}^{\mathrm{f}}$ representation of the group $\mathcal{P}$, with respect to which the SchrödingerFoldy equation (4) is invariant, are given by (24), (25) in the $\vec{x}$-realization of the space $\mathrm{H}^{3,4}(7)$ and by 


$$
\begin{gathered}
p_{0}=\omega, p_{\ell}=k_{\ell}, \widetilde{j}_{\ell n}=\widetilde{x}_{\ell} k_{n}-\widetilde{x}_{n} k_{\ell}+s_{\ell n} ; \\
\left(\widetilde{x}_{\ell}=-i \widetilde{\partial}_{\ell}, \widetilde{\partial}_{\ell} \equiv \frac{\partial}{\partial k^{\ell}}\right), \\
\widetilde{j}_{0 \ell}=-\widetilde{j}_{\ell 0}=t k_{l}-\frac{1}{2}\left\{\widetilde{x}_{\ell}, \omega\right\}-\left(\frac{s_{\ell n} k_{n}}{\omega+m} \equiv \breve{s}_{\ell}\right),
\end{gathered}
$$

in the momentum $\vec{k}$-realization $\widetilde{\mathrm{H}}^{3,4}$ of the doublet states space, respectively. The explicit form of the spin terms $s_{l n}$ in the equations $(24),(25),(31),(32)$, which is used for the $e^{-} e^{+}$-doublet, is given in the definition (29).

Despite the manifestly non-covariant forms (24), (25), (31), (32) of the $\mathcal{P}^{\mathrm{f}}$-generators, they satisfy the commutation relations of the $\mathcal{P}$ algebra in the manifestly covariant form (3).

The $\mathcal{P}^{\mathrm{f}}$-representation of the group $\mathcal{P}$ in the space $\mathrm{H}^{3,4}(7)$ is given by a converged in this space exponential series $(23)$ or, in the momentum space $\widetilde{\mathrm{H}}^{3,4}$, by a corresponding exponential series given in terms of the generators (31), (32).

The corresponding Casimir operators have the form

$$
\begin{gathered}
p^{2}=\widehat{p}^{\mu} \widehat{p}_{\mu}=m^{2} \mathrm{I}_{4}, \\
W=w^{\mu} w_{\mu}=m^{2} \vec{s}^{2}=\frac{1}{2}\left(\frac{1}{2}+1\right) m^{2} \mathrm{I}_{4},
\end{gathered}
$$

where $\vec{s}$ is given in (29) and $\mathrm{I}_{4}$ is $4 \times 4$ unit matrix.

We emphasize that the modern definition of $\mathcal{P}$ invariance (or $\mathcal{P}$ symmetry) of the equation of motion (4) in $\mathrm{H}^{3,4}$ is given by the following assertion, see, e. g. [23]. The set $\mathrm{F} \equiv\{f\}$ of all possible solutions of the equation (4) is invariant with respect to the $\mathcal{P}^{\mathrm{f}}$-representation of the group $\mathcal{P}$, if for arbitrary solution $f$ and arbitrarilyfixed parameters $(a, \varpi)$ the assertion

$$
(a, \varpi) \rightarrow U(a, \varpi)\{f\}=\{f\} \equiv \mathrm{F}
$$

is valid. Furthermore, the assertion (35) is ensured by the fact that (as it is easy to verify) all the $\mathcal{P}$-generators (24), (25) commute with the operator $i \partial_{t}-\sqrt{-\Delta+m^{2}}$ of the equation (4).

In spite of the fact that in RCQM many manifestly noncovariant objects are used, the model under consideration is relativistic invariant in the sense of the definition given above.

On the main and additional conservation laws.

Similarly to the nonrelativistic quantum mechanics the conservation laws are found in the form of quantummechanical mean values of the operators, which commute with the operator of the equation of motion.

The important physical consequence of the assertion about the relativistic invariance is the fact that 10 integral dynamical variables of the doublet

$$
\left(P_{\mu}, J_{\mu \nu}\right) \equiv \int d^{3} x f^{\dagger}(t, \vec{x})\left(\widehat{p}_{\mu}, \widehat{j}_{\mu \nu}\right) f(t, \vec{x})=\mathrm{Const}
$$

do not depend on time, i. e. they are the constants of motion for this doublet.

Note that the external and internal degrees of freedom for the free $e^{-} e^{+}$-doublet are independent. Therefore, the operator $\vec{s}$ (29) commutes not only with the operators $\widehat{\vec{p}}, \vec{x}$, but also with the orbital part $\widehat{m}_{\mu \nu}$ of the total angular momentum operator. And both operators $\vec{s}$ and $\widehat{m}_{\mu \nu}$ commute with the operator $i \partial_{t}-\sqrt{-\Delta+m^{2}}$ of the equation (4). Therefore, besides the 10 main (consequences of the 10 Poincaré generators) conservation laws (36), 12 additional constants of motion exist for the free $e^{-} e^{+}$-doublet. These additional conservation laws are the consequences of the operators of the following observables:

$$
\begin{gathered}
s_{j}, \breve{s}_{\ell}=\frac{s_{\ell n} \widehat{p}_{n}}{\widehat{\omega}+m}, \widehat{m}_{\ell n}=x_{l} \widehat{p}_{n}-x_{n} \widehat{p}_{\ell}, \\
\widehat{m}_{0 \ell}=-\widehat{m}_{l 0}=t \widehat{p}_{\ell}-\frac{1}{2}\left\{x_{\ell}, \widehat{\omega}\right\},
\end{gathered}
$$

where $s_{j}=s_{\ell n}$ are given in (29).

Thus, the following assertions can be proved. In the space $\mathrm{H}^{\mathrm{A}}=\{A\}$ of the quantum-mechanical amplitudes the 10 main conservation laws (36) have the form

$$
\left(P_{\mu}, J_{\mu \nu}\right)=\int d^{3} k A^{\dagger}(\vec{k})\left(\widetilde{p}_{\mu}, \widetilde{j}_{\mu \nu}\right) A(\vec{k}), A(\vec{k}) \equiv\left|\begin{array}{c}
a_{\mathrm{r}}^{-} \\
a_{\dot{\mathrm{r}}}^{+}
\end{array}\right|,
$$

where the density generators of $\mathcal{P}^{\mathrm{A}},\left(\widetilde{p}_{\mu}, \widetilde{j}_{\mu \nu}\right)$ of (38) are given by

$$
\widetilde{p}_{0}=\omega, \widetilde{p}_{l}=k_{l}, \widetilde{j}_{l n}=\widetilde{x}_{l} k_{n}-\widetilde{x}_{n} k_{l}+s_{l n} ; \quad\left(\widetilde{x}_{l}=-i \frac{\partial}{\partial k^{l}}\right),
$$

$$
\widetilde{j}_{0 l}=-\widetilde{j}_{l 0}=-\frac{1}{2}\left\{\widetilde{x}_{l}, \omega\right\}-\left(\breve{s}_{l} \equiv \frac{s_{l n} k_{n}}{\omega+m}\right) .
$$

In the formula $(38) A(\vec{k}) \equiv\left|\begin{array}{c}a_{\mathrm{r}}^{-} \\ a_{\hat{r}}^{+}\end{array}\right|$is a 4-column of amplitudes $a_{+}^{-}(\vec{k}), a_{-}^{-}(\vec{k}), a_{-}^{+}(\vec{k}), a_{+}^{+}(\vec{k})$, where $\mathrm{r}=$ $(+.-) \cdot \dot{r}=(-,+)$.

Note that the operators (38)-(40) satisfy the Poincaré commutation relations in the manifestly covariant form (3).

It is evident that the 12 additional conservation laws

$$
\left(M_{\mu \nu}, S_{\ell n}, \breve{S}_{\ell}\right) \equiv \int d^{3} x f^{\dagger}(t, \vec{x})\left(\widehat{m}_{\mu \nu}, s_{\ell n}, \breve{s}_{\ell}\right) f(t, \vec{x})
$$

generated by the operators (37), are the separate terms in the expressions (38)-(40) of principal (main) conservation laws.

\section{On the Clifford-Dirac algebra.}

The Clifford-Dirac algebra of the $\gamma$-matrices must be introduced into the $\mathrm{FW}$ representations. The reasons are as follows.

Part of the Clifford-Dirac algebra operators are directly related to the spin $1 / 2$ doublet operators $\left(\frac{1}{2} \gamma^{2} \gamma^{3}, \frac{1}{2} \gamma^{3} \gamma^{1}, \frac{1}{2} \gamma^{1} \gamma^{2}\right)$. In the FW representation for the spinor field these spin operators commute with the Hamiltonian and with the operator of the equation of 
motion (11). In the Pauli-Dirac representation these operators do not commute with the Dirac equation operator. Only the sums of the orbital operators and such spin operators commute with the Diracian. So if we want to relate the orts of the Clifford-Dirac algebra with the actual spin we must introduce this algebra into the $F W$ representation.

In the quantum-mechanical representation (i. e. in the space of the solutions (8) of the Schrödinger-Foldy equation(4)) the $\gamma$-matrices are obtained by the transformation $v$ given in the formula (43) below.

Moreover, we use the generalized Clifford-Dirac algebra over the field of real numbers. This algebra was introduced in Refs. [12-16]. The use of 29 orts of this proper extended real Clifford-Dirac algebra gives the additional possibilities in comparison with only 16 elements of the standard Clifford-Dirac algebra, see, e. g., [12-16].

The definitions of spin matrices (29) de facto determine a so-called "quantum-mechanical" representation of the Dirac matrices

$$
\bar{\gamma}^{\mu}: \bar{\gamma}^{\mu} \bar{\gamma}^{\nu}+\bar{\gamma}^{\nu} \bar{\gamma}^{\mu}=2 g^{\mu \nu} ; \bar{\gamma}_{0}^{-1}=\bar{\gamma}_{0}, \bar{\gamma}_{l}^{-1}=-\bar{\gamma}_{l}
$$

The matrices $\bar{\gamma}^{\mu}(42)$ of this representation are linked to the Dirac matrices $\gamma^{\mu}$ in the standard Pauli-Dirac (PD) representation:

$$
\begin{array}{r}
\bar{\gamma}^{0}=\gamma^{0}, \bar{\gamma}^{1}=\gamma^{1} C, \bar{\gamma}^{2}=\gamma^{0} \gamma^{2} C, \bar{\gamma}^{3}=\gamma^{3} C, \bar{\gamma}^{4}=\gamma^{0} \gamma^{4} C \\
\bar{\gamma}^{\mu}=v \gamma^{\mu} v, \quad v \equiv\left|\begin{array}{ll}
\mathrm{I}_{2} & 0 \\
0 & C \mathrm{I}_{2}
\end{array}\right|=v^{-1}
\end{array}
$$

where the standard Dirac matrices $\gamma^{\mu}$ are given by

$\gamma^{0}=\left|\begin{array}{ll}\mathrm{I}_{2} & 0 \\ 0 & -\mathrm{I}_{2}\end{array}\right|, \quad \gamma^{k}=\left|\begin{array}{ll}0 & \sigma^{k} \\ -\sigma^{k} & 0\end{array}\right|, \quad \mu=0,1,2,3$

Note that in the terms of $\bar{\gamma}^{\mu}$ matrices (43) the spin operator (29) have the form $\vec{s}=\frac{i}{2}\left(\bar{\gamma}^{2} \bar{\gamma}^{3}, \bar{\gamma}^{3} \bar{\gamma}^{1}, \bar{\gamma}^{1} \bar{\gamma}^{2}\right)$.

The $\bar{\gamma}^{\mu}$ matrices (43) together with the matrix $\bar{\gamma}^{4} \equiv$ $\bar{\gamma}^{0} \bar{\gamma}^{1} \bar{\gamma}^{2} \bar{\gamma}^{3}$, imaginary unit $i \equiv \sqrt{-1}$ and operator $C$ of complex conjugation in $\mathrm{H}^{3,4}$ generate the quantummechanical representations of the extended real CliffordDirac algebra and proper extended real Clifford-Dirac algebra, which were put into consideration in [12] (see also $[13-16])$.

On the principles of heredity and the correspondence. The explicit forms (36)-(41) of the main and additional conservation laws demonstrate evidently that the model of RCQM satisfies the principles of the heredity and the correspondence with the nonrelativistic classical and quantum theories. The deep analogy between RCQM and these theories for the physical system with the finite number degrees of freedom (where the values of the free dynamical conserved quantities are additive) is also evident.

Our new way of the Dirac equation derivation (section 9 below) is started from the RCQM of the spin $1 / 2$ doublet, which is formulated above.

\section{The second quantization}

Finally, we consider briefly the program of the canonical quantization of the RCQM model. Note that the expression for the total energy $P_{0}$ plays a special role in the procedure of a so called "second quantization". In the RCQM doublet model, as it is evident from the expression for the $P_{0}$ in (38) in terms of the charge signmomentum-spin amplitudes

$$
P_{0}=\int d^{3} k \omega\left(\left|a_{\mathrm{r}}^{-}(\vec{k})\right|^{2}+\left|a_{\hat{\mathrm{r}}}^{+}(\vec{k})\right|^{2}\right) \geq m>0,
$$

the energy is positive. The same assertion is valid for the amplitudes related to the arbitrary-fixed stationary complete set of operators. Furthermore, the operator $\widehat{P}_{0}$ of the quantized energy corresponding to expression (45) is a positive-valued operator. The explicit form of the operator $\widehat{P}_{0}$ follows from the expression (45) after the anticommutation quantization of the amplitudes

$$
\left\{\widehat{a}_{\alpha}(\vec{k}), \widehat{a}_{\beta}^{\dagger}(\vec{k})\right\}=\delta_{\alpha \beta} \delta\left(\vec{k}-\vec{k}^{\prime}\right)
$$

(other operators anticommute) and their substitution $a^{\mp} \rightarrow \widehat{a}^{\mp}$ into the formula (45). Note that the quantized amplitudes determine the Fock space $\mathcal{H}^{\mathrm{F}}$ (over the quantum-mechanical space $\mathrm{H}^{3,4}$ ). Moreover, the operators of dynamical variables $\widehat{P}_{\mu}, \widehat{J}_{\mu \nu}$ in $\mathcal{H}^{\mathrm{F}}$, which are expressed according to formulae (38) in terms of the operator amplitudes $\widehat{a}_{\alpha}(\vec{k}), \widehat{a}_{\beta}^{\dagger}(\vec{k})$, automatically have the form of "normal products" and satisfy the commutation relations (3) of the $\mathcal{P}$ group in the Fock space $\mathcal{H}^{\mathrm{F}}$. Operators $\widehat{P}_{\mu}, \widehat{J}_{\mu \nu}$ determine the corresponding unitary representation in $\mathcal{H}^{\mathrm{F}}$. Other details are not the subject of this paper.

\section{Brief review of the different ways of the Dirac equation derivation}

Below a brief review of different ways of the Dirac equation derivation is given.

One should note the elegant derivation given by Paul Dirac in his book [24]. Until today it is very interesting for the readers to feel Dirac's way of thinking and to follow his logical steps. Nevertheless, the Dirac's consideration of the Schrödinger-Foldy equation, which was essentially used in his derivation [24], was not correct. Especially his assertion that Schrödinger-Foldy equation is unsatisfactory from the point of view of the relativistic theory. Dirac's doubts were overcomed in Ref. [1-3]. Today it is confirmed by more then a hundred publications about FW and the spinless Salpeter equations, which have wide-range application in contemporary theoretical physics.

In the well-known book [25] one can find excellent review of the Dirac theory and two different ways of the Dirac equation derivation. First, it is the presentation of the Klein-Gordon equation in the form of a first-order differential system of equations, factorization of the Klein-Gordon operator. Second, the Lagrange 
approach is considered and the Dirac equation is derived from the variational Euler-Lagrange least action principle.

In van der Waerden-Sakurai derivation [26] of the Dirac equation the spin of the electron is incorporated into the nonrelativistic theory. The representation of the nonrelativistic kinetic energy operator of the free spin $1 / 2$ particle in the form $H^{\mathrm{KE}}=(\vec{\sigma} \cdot \vec{p})(\vec{\sigma} \cdot \vec{p}) / 2 m$ and the relativistic expression $E^{2}-\vec{p}^{2}=m^{2}$ are used. Then the procedure of transition from 2-component to 4-component equation is fulfilled and explained.

In the book [27] (second edition) the Dirac equation is derived from the manifestly covariant transformational properties of the 4-component spinor.

The derivation of the Dirac equation from the initial geometric properties of the space-time and electron together with wide-range discussion of the geometric principles of the electron theory is the main content of the book [28]. The ideas of V. Fock and D. Iwanenko [29, $30]$ on the geometrical sense of Dirac $\gamma$-matrices are the basis of the approach.

One should point out the derivation of the Dirac equation based on the Bargman-Wigner classification of the irreducible unitary representations of the Poincaré group, see e. g. [31]. It is an illustrative demonstration of the possibilities of the group-theoretical approach for the elementary particle physics.

In L. Foldy's papers [1-3] one can easy find the reverse problem, in which the Dirac equation is obtained from the FW equation. Nevertheless, it is only the transition from one representation of the spinor field to another.

H. Sallhofer [32, 33] derived the Dirac equation for hydrogen spectrum starting from the Maxwell equations in medium. Strictly speaking, only the stationary equations were considered.

In Ref. [34] quaternion measurable processes were introduced and the Dirac equation was derived from the Langevin equation associated with a two-valued process.

The author of Ref. [35] was able to derive the Dirac equation from the conservation law of spin $1 / 2$ current. The requirement that this current is conserved leads to a unique determination of the Lorentz invariant equation satisfied by the relativistic spin $1 / 2$ field. Let us briefly comment that the complete list of conservation laws for the Dirac theory is the Noether consequence of the Dirac equation. Therefore, the validity of the inverse problem is really expected. Can it be considered an independent derivation?

The Dirac equation was derived [36] from the master equation of Poisson process by analytic continuation. The extension to the case where a particle moves in an external field was given. It was shown that the generalized master equation is closely related to the threedimensional Dirac equation in an external field.

In Ref. [37], a method of deriving the Dirac equation from the relativistic Newton's second law was suggested. Such derivation is possible in a new formalism, which relates the special form of relativistic mechanics to the quantum mechanics. The author suggested a concept of a velocity field. At first, the relativistic Newton's second law was rewritten as a field equation in terms of the velocity field, which directly reveals a new relationship linked to the quantum mechanics. After that it was shown that the Dirac equation can be derived from the field equation in a rigorous and consistent manner.

A geometrical derivation of the Dirac equation, by considering a spin $1 / 2$ particle traveling with the speed of light in a cubic spacetime lattice, was made in Ref. [38]. The mass of the particle acts to flip the multicomponent wavefunction at the lattice sites. Starting with a difference equation for the case of one spatial and one time dimensions, the authors generalize the approach to higher dimensions. Interactions with external electromagnetic and gravitational fields are also considered. Nevertheless, the idea of such derivation is based on the Dirac's observation that the instantaneous velocity operators of the spin $1 / 2$ particle (hereafter called by the generic name the electron) have eigenvalues $\pm c$. This mistake of Dirac was demonstrated and overcome in Ref. [1].

Using the mathematical tool of Hamilton's biquaternions, the authors of Ref. [39] propose a derivation of the Dirac equation from the geodesic equation. Such derivation is given in the program of application of the theory of scale relativity to the purposes of microphysics at recovering quantum mechanics as a new non-classical mechanics on a non-derivable space-time.

M. Evans was successful to express his equation of general relativity (generally covariant field equation for gravitation and electromagnetism [40]) in spinor form, thus producing the Dirac equation in general relativity [41]. The Dirac equation in special relativity is recovered in the limit of Euclidean or flat spacetime.

Ten years ago we already presented our own derivation of the Dirac equation [42-44]. The Dirac equation was derived from slightly generalized Maxwell equations with gradient-like current and charge densities. This form of the Maxwell equations, which is directly linked with the Dirac equation, is the maximally symmetrical variant of these equations. Such Maxwell equations are invariant with respect to a 256-dimensional algebra (the well-known algebra of conformal group has only 15 generators). Of course, we derived only massless Dirac equation.

Below we present a new derivation of the Dirac equation. We derive the Dirac equation from the 4-component Schrödinger-Foldy equation (4) of the RCQM. The RCQM model of the spin $1 / 2$ particle doublet is formulated here in Section 6. Hence, we derive the Dirac equation from the more fundamental model of the spin $1 / 2$ particle doublet.

\section{Derivation of the Foldy- Wouthuysen and the standard Dirac equations}

At first we demonstrate that the FW and the standard Dirac equations are direct and unambiguous consequences of the Schrödinger-Foldy equation (4) and of the RCQM model. Further we consider briefly the physical and mathematical sense of three different models of the fermionic doublet (RCQM, the FW model, the Dirac model) at the example of comparison of the grouptheoretical approaches to these three models. 
Thus, below we search for the infallible links between the RCQM, the FW model and the Dirac model of the fermionic doublet. The links between the objects of equations (and between the associated operators of the algebra of observables) of these three models are under consideration. The following mathematical and physical assertions should be taken into account in order to perform such a program.

The Poincaré group $\mathcal{P}$ is the group of real parameters $(a, \varpi)$, i. e. it is a real Lie group. Therefore, as a matter of fact the prime (anti-Hermitian) generators

$$
\left(p_{\mu}, j_{\mu \nu}\right)^{\text {prime }} \equiv\left(p_{\mu}^{\mathrm{pr}}, j_{\mu \nu}^{\mathrm{pr}}\right) \equiv\left(-i p_{\mu},-i j_{\mu \nu}\right)
$$

are the generators of the group $\mathcal{P}$, and not the operators $\left(p_{\mu}, j_{\mu \nu}\right)$, which were used in all the above formulae. In terms of prime generators $(47)$ the $\mathcal{P}$-representation in the space $\mathrm{H}^{3,4}$ is given by the formula

$$
(a, \varpi) \rightarrow U(a, \varpi)=\exp \left(a^{\mu} p_{\mu}^{\mathrm{pr}}+\frac{1}{2} \varpi^{\mu \nu} j_{\mu \nu}^{\mathrm{pr}}\right),
$$

and the commutation relations of the Lie algebra of the group $\mathcal{P}$ in manifestly covariant form are given by

$$
\begin{array}{r}
{\left[p_{\mu}^{\mathrm{pr}}, p_{\nu}^{\mathrm{pr}}\right]=0, \quad\left[p_{\mu}^{\mathrm{pr}}, j_{\rho \sigma}^{\mathrm{pr}}\right]=g_{\mu \rho} p_{\sigma}-g_{\mu \sigma} p_{\rho}} \\
{\left[j_{\mu \nu}^{\mathrm{pr}}, j_{\rho \sigma}^{\mathrm{pr}}\right]=-g_{\mu \rho} j_{\nu \sigma}^{\mathrm{pr}}-g_{\rho \nu} j_{\sigma \mu}^{\mathrm{pr}}-g_{\nu \sigma} j_{\mu \rho}^{\mathrm{pr}}-g_{\sigma \mu} j_{\rho \nu}^{\mathrm{pr}}}
\end{array}
$$

It was demonstrated [12-16] that the prime (antiHermitian) generators play a special role in the grouptheoretical approach to the quantum theory and symmetry analysis of the corresponding equations. It is the use of the anti-Hermitian generators of the groups under consideration that allowed us $[12-16]$ to find additional bosonic properties of the FW and Dirac equations. The mathematical correctness of appealing to the antiHermitian generators is considered in details in Refs. $[45,46]$.

Below in all cases we use only the prime (antiHermitian) $\mathcal{P}$-generators (and the prime-operators of all energy-momentum an angular momentum quantities are used). Therefore, after this warning in the consideration below the notation "prime" or "pr" of the corresponding operators is omitted.

Moreover, we rewrite the explicit forms of the Schrödinger-Foldy, the FW and the Dirac equations in the terms of anti-Hermitian operators.

The link between the Schrödinger-Foldy equation (4) and the FW equation (11) is given by the operator $v$

$$
v=\left|\begin{array}{ll}
\mathrm{I}_{2} & 0 \\
0 & C \mathrm{I}_{2}
\end{array}\right| ; \quad v^{2}=\mathrm{I}_{4}, \quad \mathrm{I}_{2}=\left|\begin{array}{ll}
1 & 0 \\
0 & 1
\end{array}\right|,
$$

(we mentioned the existence of such an operator in the formulae (43)), $C$ is the operator of complex conjugation, the operator of involution in the space $\mathrm{H}^{3,4}$. For the non-singular operator $v$ from (50) the equality

$$
v\left(\partial_{0}+i \widehat{\omega}\right) v=\partial_{0}+i \gamma^{0} \widehat{\omega}
$$

is valid. It means that after the transformation $f \rightarrow \phi=$ $v f$ the Schrödinger-Foldy equation (4) becomes the FW equation (11)

$$
\left(\partial_{0}+i \gamma^{0} \widehat{\omega}\right) \phi(x)=0 ; \quad \phi=v f \equiv\left|\begin{array}{c}
\varphi_{-} \\
\varphi_{+}^{*}
\end{array}\right| \in \mathrm{H}^{3,4} .
$$

The inverse transformation is also valid

$$
f=v \phi .
$$

The operator $v(50)$ transforms arbitrary operator $q$ of the RCQM into the operator $Q$ in the FW representation for the spinor field and vice versa:

$$
Q=v q v \leftrightarrow q=v Q v
$$

The only warning is that formula (54) is valid only for the anti-Hermitian operators! It means that in order to avoid mistakes one should apply this formula only for the prime (anti-Hermitian) energy-momentum, angular momentum and spin quantities. Especially if one uses the operator (50) for the transformation of the commutation relations.

The Foldy-Wouthuysen model of the fermionic doublet. Comparison of the RCQM and the FW model of the fermionic doublet is the content of the section 4 . Here we consider some additional important features of the FW model.

The quantum-mechanical sense of the object $\phi$ is the following. The equation (52) is a system of two 2component equations

$$
\left(\partial_{0}+i \widehat{\omega}\right) \varphi_{-}(x)=0, \quad\left(\partial_{0}-i \widehat{\omega}\right) \varphi_{+}^{*}(x)=0 .
$$

The first equation is the equation for the wave function $\varphi$ - of the electron and the second is the one for the function $\varphi_{+}^{*}$ being the complex conjugate to the wave function $\varphi_{+}$of the positron.

Under the transformations (54) the $\mathcal{P}^{\mathrm{f}}$-generators (24), (25) (taken with the spin term (29) and in the prime anti-Hermitian form) become the prime $\mathcal{P}^{\phi_{-}}$ generators $(\mathcal{P}$-symmetries of the $\mathrm{FW}$ equation $(52))$

$\widehat{p}_{0}=-i \gamma_{0} \widehat{\omega}, \widehat{p}_{\ell}=\partial_{\ell}, \widehat{j}_{\ell n}=x_{\ell} \partial_{n}-x_{n} \partial_{\ell}+\widehat{s}_{\ell n} \equiv \widehat{m}_{\ell n}+\widehat{s}_{\ell n}$,

$$
\widehat{j}_{0 \ell}=t \partial_{\ell}+\frac{i}{2} \gamma_{0}\left\{x_{l}, \widehat{\omega}\right\}+\gamma_{0} \frac{\widehat{s}_{\ell n} \widehat{p}_{n}}{\widehat{\omega}+m}
$$

where

$$
\widehat{s}_{\ell n}=\widehat{s}^{\ell n} \equiv \frac{1}{4}\left[\gamma^{\ell}, \gamma^{n}\right],
$$

and $\gamma^{\mu}$ are the standard Dirac matrices (44) (in the Pauli-Dirac representation).

Therefore, the $\mathcal{P}^{\phi}$-generators $(56),(57)$ of the $\mathcal{P}^{\phi}$ representation in $\mathrm{H}^{3,4}$ (as well as the operators $q^{\phi}=$ $v q^{\mathrm{f}} v$ ) of the algebra of all observable physical quantities in the FW model of the fermionic doublet) are the functions generated by the 10 operators

$$
\vec{x}=\left(x^{j}\right) \in \mathrm{R}^{3}, \widehat{\vec{p}}=-\nabla, \widehat{\vec{s}} \equiv\left(\widehat{s}_{23}, \widehat{s}_{31}, \widehat{s}_{12}\right), \gamma^{0},
$$

where $\widehat{s}_{\ell n}$ are given in (58). The physical sense of these operators (as well as of the functions $q^{\phi}$ from them) 
follows from the physical sense of the corresponding quantum-mechanical operators $\left(\vec{x}, \vec{p} \vec{s}, \gamma^{0}\right)$ (and $\left.q^{\mathrm{f}}\right)$, which are verified by the principles of the heredity and correspondence with non-relativistic quantum and classical theories.

The solution (8) of the SF equation (4), which is associated with the stationary complete set of operators (momentum - sign of the charge - spin projection $\bar{s}_{\mathrm{z}}$ ) is transformed into the solution (12) of the FW equation (11), where $a_{+}^{-}(\vec{k}), a_{-}^{-}(\vec{k})$ are the same as in $(8)$ and $a_{-}^{*+}(\vec{k}), a_{+}^{*+}(\vec{k})$ are the complex conjugated functions to the quantum-mechanical amplitudes $a_{-}^{+}(\vec{k}), a_{+}^{+}(\vec{k})$ of the standard quantum-mechanical sense, which was considered in Section 6 .

Further, similarly to the RCQM in the FW model the additional conservation laws also exist together with the main 10 Poincaré conservation quantities

$$
\left(\widehat{p}_{\mu}, \widehat{j}_{\mu \nu}\right)^{\phi} \rightarrow\left(P_{\mu}, J_{\mu \nu}\right)^{\phi}=\int d^{3} x \phi^{\dagger}(x) i\left(\widehat{p}_{\mu}, \widehat{j}_{\mu \nu}\right)^{\phi} \phi(x)
$$

The 12 additional conservation laws, which were considered in Section 2, also exist and can be very easy calculated here. Naturally, due to non-unitarity of the operator $v$ from (50) the explicit form of the conservation laws (60) does not coincide with the quantummechanical quantities (36). It is evident from the expression (60) in the terms of quantum-mechanical amplitudes

$$
\left(P_{\mu}, J_{\mu \nu}\right)^{\phi}=\int d^{3} k A^{\phi \dagger}(\vec{k})\left(\widetilde{p}_{\mu}, \widetilde{j}_{\mu \nu}\right)^{\phi} A^{\phi}(\vec{k})
$$

where $A^{\phi}(\vec{k})$ has the form $A^{\phi}(\vec{k}) \equiv\left|\begin{array}{c}a_{+}^{-} \\ a_{-}^{-} \\ a_{-}^{*+} \\ a_{+}^{*+}\end{array}\right|$,

$\left(\widetilde{p}_{\mu}, \widetilde{j}_{\mu \nu}\right)^{\phi}$ are given by

$$
\begin{gathered}
\widetilde{p}_{0}=\gamma^{0} \omega, \widetilde{p}_{\ell}=\gamma^{0} k_{\ell}, \widetilde{j}_{\ell n}=\widetilde{x}_{\ell} k_{n}-\widetilde{x}_{n} k_{\ell}+\widehat{s}_{\ell n}, \\
\widetilde{j}_{0 \ell}=-\widetilde{j}_{\ell 0}=-\frac{1}{2}\left\{\widetilde{x}_{\ell}, \omega\right\}+\gamma^{0}\left(\widetilde{\widetilde{s}}_{\ell} \equiv \frac{\widehat{s}_{\ell n} k_{n}}{\omega+m}\right),
\end{gathered}
$$

and the definitions $\left(\widetilde{x}_{\ell}=-i \widetilde{\partial}_{\ell}, \widetilde{\partial}_{\ell} \equiv \frac{\partial}{\partial k^{\ell}}\right), \omega \equiv$ $\sqrt{\vec{k}^{2}+m^{2}}$ are used.

For example, the total energy of the field $\phi$, instead of the expression (45) in RCQM, has the form

$$
P_{0}=\int d^{3} k \omega\left(a_{\mathrm{r}}^{*-}(\vec{k}) a_{\mathrm{r}}^{-}(\vec{k})-a_{\hat{\mathrm{r}}}^{+}(\vec{k}) a_{\hat{\mathrm{r}}}^{*+}(\vec{k})\right),
$$

which is not positively defined. In this sense, the FW model (whose quantum-mechanical content is unambiguous) in fact is not the quantum-mechanical model for the $e^{-} e^{+}$-doublet. Therefore, in the procedure of "canonical quantization" of the field $\phi$ on the basis of anticommutation relations (46) an additional axiom is applied in this model for the quantized field $\widehat{\phi}$.
According to this axiom the definition of the operators of the physical quantities in the Fock space $\mathcal{H}^{\mathrm{F}}$ is extended by taking them only in the form of "normal products" with respect to the operator amplitudes $\widehat{a}_{\mathrm{r}}^{-}(\vec{k}), \widehat{a}_{\mathrm{r}}^{*-}(\vec{k}), \widehat{a}_{\hat{\mathrm{r}}}^{+}(\vec{k}), \widehat{a}_{\dot{\mathrm{r}}}^{*+}(\vec{k})$. It is easy to verify that the operators of 10 main conserved quantities of the "quantized field" $\widehat{\phi}$ in the form of normal products coincide with the corresponding expressions in the "second quantized" RCQM model of the Fermionic doublet

$$
:\left(\widehat{P}_{\mu}, \widehat{J}_{\mu \nu}\right)^{\phi}:=\int d^{3} k \widehat{A}^{\dagger}(\vec{k})\left(\widetilde{p}_{\mu}, \widetilde{j}_{\mu \nu}\right) \widehat{A}(\vec{k}),
$$

where $\widehat{A}(\vec{k}) \equiv\left|\begin{array}{c}\widehat{a}_{+}^{-} \\ \widehat{a}_{-}^{-} \\ \widehat{a}_{-}^{+} \\ \widehat{a}_{+}^{+}\end{array}\right|$and $\left(\widetilde{p}_{\mu}, \widetilde{j}_{\mu \nu}\right)$ are given in (39), (40).

The Dirac model of the fermionic doublet. Taking into account the above consideration and using the well-known from Ref. [1] transition operators $V^{ \pm}$

$\phi \rightarrow \psi=V^{+} \phi, \psi \rightarrow \phi=V^{-} \psi, \quad V^{ \pm} \equiv \frac{ \pm i \gamma^{l} \partial_{l}+\widehat{\omega}+m}{\sqrt{2 \widehat{\omega}(\widehat{\omega}+m)}}$

we find the resulting operator

$W=V^{+} v, W^{-1}=v V^{-}: f \rightarrow \psi=W f, \psi \rightarrow f=W^{-1} \psi$,

$W W^{-1}=W^{-1} W=1$, which transforms (one-to-one) all quantities of RCQM model into the corresponding quantities of the Dirac model and vice versa. For example,

$$
W\left(\partial_{0}+i \widehat{\omega}\right) W^{-1}=\partial_{0}+i H_{\mathrm{D}} ; \quad H_{\mathrm{D}} \equiv \vec{\alpha} \cdot \vec{p}+\beta m
$$

It means that quantum-mechanical Schrödinger-Foldy equation (4) (in the anti-Hermitian form (51)) is transformed into the Dirac equation in the Schrödinger form

$$
\begin{gathered}
\left(\partial_{0}+i \gamma^{0} \widehat{\omega}\right) \phi(t, \vec{x})=0 \rightarrow \\
{\left[\partial_{0}+i(\vec{\alpha} \cdot \vec{p}+\beta m)\right] \psi(t, \vec{x})=0 .}
\end{gathered}
$$

Furthermore,

$$
W\left(\widehat{p}_{\mu}, \widehat{j}_{\mu \nu}\right) W^{-1}=\left(\breve{p}_{\mu}, \breve{j}_{\mu \nu}\right)^{\text {Dirac }}
$$

where

$\breve{p}_{0}=-i H_{\mathrm{D}}, \breve{p}_{\ell}=\partial_{\ell}, \breve{j}_{\ell n}=x_{\ell} \partial_{n}-x_{n} \partial_{\ell}+\widehat{s}_{\ell n} \equiv \widehat{m}_{\ell n}+\widehat{s}_{\ell n}$,

$\breve{j}_{0 \ell}=t \partial_{\ell}+\frac{i}{2}\left\{x_{l}, H_{\mathrm{D}}\right\}=t \partial_{\ell}-x_{\ell} \breve{p}_{0}+\widehat{s}_{0 \ell} ; \quad \widehat{s}_{\mu \nu} \equiv \frac{1}{4}\left[\gamma_{\mu}, \gamma_{\nu}\right]$

Note that the generators of the local $\mathcal{P}^{\psi}$ representation (the standard $\mathcal{P}$-algebra of invariance of the Dirac equation (69)) have the form

$$
p_{\mu}=\partial_{\mu}, \quad j_{\mu \nu}=x_{\mu} \partial_{\nu}-x_{\nu} \partial_{\mu}+\widehat{s}_{\mu \nu} .
$$


Moreover, in the manifold $\psi \subset \mathrm{H}^{3,4}$ of the solutions of the Dirac equation (69) operators (73) coincide with the $\mathcal{P}^{\mathrm{I}}$-generators $(71),(72)$. Therefore, the $\mathcal{P}^{\psi}$ representation determined by the generators (71), (72) is called induced (I). As a consequence of this fact, for example, the following equalities are valid for the 10 main dynamical variables of the field $\psi$

$$
\begin{gathered}
\left(p_{\mu}, j_{\mu \nu}\right) \rightarrow\left(P_{\mu}, J_{\mu \nu}\right)^{\psi} \equiv \\
\int d^{3} x \bar{\psi} i\left(p_{\mu}, j_{\mu \nu}\right) \psi=\int d^{3} x \bar{\psi} i\left(\breve{p}_{\mu}, \breve{j}_{\mu \nu}\right) \psi=\left(P_{\mu}, J_{\mu \nu}\right)^{\phi},
\end{gathered}
$$

where the conservation laws $\left(P_{\mu}, J_{\mu \nu}\right)^{\phi}$ are given by the formula (60) and in terms of amplitudes $a_{\mathrm{r}}^{-}(\vec{k}), a_{\mathrm{r}}^{*-}(\vec{k}), a_{\hat{\mathrm{r}}}^{+}(\vec{k}), a_{\mathrm{r}}^{*+}(\vec{k})$ are given by the formula (61). It means that the procedure of canonical quantization of the field $\psi$ is reduced to the corresponding procedure of the field $\phi$ quantization.

We emphasize that $\mathcal{P}$-operators $(73)$ are the functions of 14 independent "generated" operators $x_{\mu}, \partial_{\mu}, \widehat{s}_{\mu \nu}$. Further, $\mathcal{P}$-generators (71), (72) are the functions of 12 independent operators $x_{\ell}, \partial_{\ell}, \widehat{s}_{\mu \nu}$. Nevertheless, only the operator $\vec{p}=-\nabla$ has the physical sense of the quantum-mechanical Fermi doublet momentum operator among the above mentioned 14 independent operators. As it was proved in Ref. [1] the operators $\vec{x}=\left(x^{\ell}\right)$ and $\widehat{s}_{\mu \nu}$, which are essentially used in the constructions (71)-(74), do not have the physical sense of the quantum-mechanical operators of the Fermionic doublet coordinate and SU(2)-spin. This fact evidently demonstrates the validity of the assertion that the standard Dirac local model is not the quantum-mechanical model of the fermionic doublet at all.

The axioms of Section 6 eventually need to be reconciled with three levels of description used in this paper: RCQM, FW and Dirac equations. Nevertheless, this interesting problem cannot be considered in few pages. Even the simple level comparison of RCQM, FW and Dirac models given above is very brief and not complete. Only the main features are considered briefly. We hope to consider these problems in few upcoming big volume papers of a review character. The readers of this paper can compare the axioms of RCQM (Section 6) with the main principles of the Dirac model, which are given in B. Thaller's monograph [9] on the high mathematical level.

\section{Few words on the discussion around antiparticle negative mass}

We emphasize that the model of the RCQM of the Fermi-doublet does not need the application of the positron negative mass concept [47-50]. It is natural due to the following reasons.

It is only the energy which depends on the mass. And the total energy together with the momentum is related to the external degrees of freedom, which are common and the same for the particle and antiparticle (for the electron and positron). The difference between $e_{-}$and $e_{+}$consists only in internal degrees of freedom such as the spin $\vec{s}$ and the sign of the charge $g=-\gamma^{0}$. Thus, if in the RCQM the mass of the particle is taken positive then the mass of the antiparticle must be taken positive too.

On the other hand, a comprehensive analysis [48] of the Dirac equation for the doublet had led the authors of Ref. [48] to the concept of the negative mass of the antiparticle. Therefore, our consideration in the previous paragraph gives the additional arguments that the Dirac model (or the Foldy-Wouthuysen model related to it) is not the quantum-mechanical one. Furthermore, in the problem of the relativistic hydrogen atom the use of the negative-frequency part $\psi^{-}(x)=e^{-i \omega t} \psi(\vec{x})$ of the spinor $\psi(x)$ in the "role of the quantum-mechanical object" is not valid. In this case neither $|\psi(\vec{x})|^{2}$, nor $\bar{\psi}(\vec{x}) \psi(\vec{x})$ is the probability distribution density with respect to the eigen values of the Fermi-doublet coordinate operator. It is due to the fact [1] that in the Dirac model the $\vec{x}$ is not the experimentally observable Fermi-doublet coordinate operator.

The application of the RCQM can be useful for the analysis of the experimental situation found in Ref. [51]. Such analysis is interesting due to the fact that (as it is demonstrated here in Sections 4-6 and 9) the RCQM is the most fundamental model of the Fermi-doublet.

Another interesting application of the RCQM is inspired by Ref. [52], where the quantum electrodynamics is reformulated in the $\mathrm{FW}$ representation. The author of Ref. [52] essentially used the result of Ref. [48] on the negative mass of the antiparticle. Starting from the RCQM we are able not to appeal to the concept of the antiparticle negative mass.

\section{Conclusions}

The model of relativistic canonical quantum mechanics on the level of axiomatic approaches to the quantum field theory is considered. The main intuitive physical principles, reinterpreted on the level of modern physical methodology, are mapped mathematically correctly into the basic assertions (axioms) of the model. The Einstein's principle of relativity is mapped as a requirement of special relativity. The principles of heredity and correspondence of the model with respect to the nonrelativistic classical and quantum mechanics are supplemented by the clarifications of external and internal degrees of freedom carriers. The principle of relativity of the model with respect to the means of cognition is implemented by the application of the rigged Hilbert space. The Schwartz test function space $\mathrm{S}^{3,4}$ is shown to be sufficient to satisfy the requirements of the principle of relativity of the model with respect to the means of cognition. And performing calculations in $\mathrm{S}^{3,4}$ does not lead to a loss of generality of the consideration.

It is shown that the algebra of experimentally observed quantities, associated with the Poincaréinvariance of the model, is determined by nine functionally independent operators $\vec{x}, \vec{p}, \vec{s}$, which in the relativistic canonical quantum mechanical model of the doublet have unambiguous physical sense. It is demonstrated that application of stationary complete sets of operators of the experimentally measured physical quan- 
tities guarantees visualization and completeness of the consideration.

In Section 8 we give a brief review of the existing ways of the Dirac equation derivation. Then, in Section 9 we consider our own method of the Dirac equation derivation.

Derivation of the Foldy-Wouthuysen and the Dirac equations from the Schrödinger-Foldy equation of relativistic canonical quantum mechanics is presented and briefly discussed. We prove that the Dirac equation is a consequence of a more elementary model of the same physical reality. The relativistic canonical quantum mechanics is suggested to be such a fundamental model of the physical reality. Moreover, it is suggested to be the most fundamental model of the Fermi spin $s=\frac{1}{2}$ doublet.

When this paper was completely ready, we have found additional references to the articles, where the problem of the derivation of the Dirac equation was considered [53-55]. Probably, this process will never end.

An important assertion is that an arbitrary physical and mathematical information, which is contained in the model of relativistic canonical quantum mechanics, is translated directly and unambiguously into the information of the same physical content in the field model of the Dirac equation.

Hence, the Dirac equation is an unambiguous consequence of the relativistic canonical quantum mechanics of the Fermi spin $s=\frac{1}{2}$-doublet (e. g., $e^{-} e^{+}$-doublet). Nevertheless, the model of relativistic canonical quantum mechanics of the Fermi-doublet has evident independent application.

\section{REFERENCES}

[1] L. Foldy, S. Wouthuysen. On the Dirac theory of spin $1 / 2$ particles and its non-relativistic limit, Phys. Rev., Vol.78, No.1, 29-36.

[2] L. Foldy. Synthesis of covariant particle equations, Phys. Rev., Vol.102, No.2, 568-581.

[3] L. Foldy. Relativistic particle systems with interaction, Phys. Rev., Vol.122, No.1, 275-288.

[4] E. Salpeter. Mass corrections to the fine structure of hydrogen-like atoms, Phys. Rev., Vol.87, No.2, 328-343.

[5] W. Lucha, F. Schobert. Relativistic Coulomb problems: Analitic upper bounds on energy levels, Phys. Rev. A., Vol.54, No.5, 3790-3794.

[6] Y. Chargui, A. Trabelsi. The zero-mass spinless Salpeter equation with a regularized inverse square potential, Phys. Lett. A., Vol.377, No.3-4, 158-168.

[7] I. Krivsky, V. Simulik, I. Lamer, T. Zajac. arXiv: 1301.6343 [math-ph] 27 Jan 2013, 17 p.
[8] I. Krivsky, V. Simulik, I. Lamer, T. Zajac. The Dirac equation as a consequence of the quantum mechanical spin 1/2 doublet model, TWMS J. App. Eng. Math., Vol.3, No.1, 62-74.

[9] B. Thaller. The Dirac equation, W.A. Benjamin Inc., Reading, Mass., 1975.

[10] I. Krivsky, V. Simulik, T. Zajac, I. Lamer. Derivation of the Dirac and Maxwell equations from the first principles of relativistic canonical quantum mechanics. Proceedings of the 14-th Internat. Conference "Mathematical Methods in Electromagnetic Theory", Institute of Radiophysics and Electronics, Kharkiv, Ukraine, 28-30 August, 2012, 201-204.

[11] N. Bogoliubov, A. Logunov, I. Todorov. Introduction to axiomatic quantum field theory, Springer-Verlag, Berlin, 1992.

[12] V. Simulik, I. Krivsky. On the extended real CliffordDirac algebra and new physically meaningful symmetries of the Dirac equation with nonzero mass, Reports of the National Academy of Sciences of Ukraine, 2010, No.5, 82-88. (in Ukrainian)

[13] I. Krivsky, V. Simulik. Fermi-Bose duality of the Dirac equation and extended real Clifford-Dirac algebra, Cond. Matt. Phys., Vol.13, No.4, 43101: 1-15.

[14] V. Simulik, I. Krivsky. Bosonic symmetries of the Dirac equation, Phys. Lett. A., Vol.375, No.25, 2479-2483.

[15] V. Simulik, I. Krivsky, I. Lamer. Application of the generalized Clifford-Dirac algebra to the proof of the Dirac equation Fermi-Bose duality, TWMS J. App. Eng. Math., Vol.3, No.1, 46-61.

[16] V. Simulik, I. Krivsky, I. Lamer. Bosonic symmetries, solutions and conservation laws for the Dirac equations with nonzero mass, Ukr. J. Phys., Vol.58, No.6, 523-533.

[17] T. Gill, W. Zachary. Analytic representation of the square-root operator, J. Phys. A., Vol.38, No.11, 2479-2496. Corrigendum. J. Phys. A., Vol.39, No.6, 1537.

[18] T. Gill, W. Zachary. M. Alfred. Analytic representation of the Dirac equation, J. Phys. A., Vol.38, No.31, 6955-6976.

[19] P. Caban, J. Rembielinski, M. Wlodarczyk. arXiv: 1308.4313 [quant-ph] 20 Aug 2013, 9 p.

[20] T. Choi. arXiv: 1209.4403 [quant-ph] 8 Jul 2013, 18 p.

[21] V. Vladimirov. Methods of the theory of generalized functions, Taylor and Francis, London, 2002. 
[22] von J. Neumann. Mathematische Grundlagen der Quantenmechanik, Verlag von Julius Springer, Berlin, 1932.

[23] G. Bluman, S. Anco. Symmetry and integration methods for differential equations, Springer, New York, 2002.

[24] P. Dirac. The principles of quantum mechanics, Clarendon Press, Oxford, 1958.

[25] N. Bogoliubov, D. Shirkov. Introduction to the theory of quantized fields, John Wiley and sons, New York, 1980 .

[26] J. Sakurai. Advanced quantum mechanics, AddisonWesley Pub. Co, New York, 1967.

[27] L. Ryder. Quantum field theory, 2-nd edit., University Press, Cambridge, 1996.

[28] J. Keller. Theory of the electron. A theory of matter from START, Kluwer Academic Publishers, Dordrecht, 2001.

[29] V. Fock, D. Iwanenko. Uber eine mogliche geometrische Deutung der relativistischen Quantentheorie, Z. Phys., Vol.54, No.11-12, 798-802.

[30] V. Fock. Geometrsierung der Diracschen Theorie des Elektrons, Z. Phys., Vol.57, No.3-4, 261-277.

[31] A. Wightman. in "Dispersion relations and elementary particles", edit. C. De Witt and R. Omnes., Wiley and Sons, New York, 1960.

[32] H. Sallhofer. Elementary derivation of the Dirac equation. I, Z. Naturforsch. A., Vol.33, 1378-1380.

[33] H. Sallhofer. Elementary derivation of the Dirac equation. X, Z. Naturforsch. A., Vol.41, 468-470.

[34] S. Strinivasan, E. Sudarshan. A direct derivation of the Dirac equation via quaternion measures, J. Phys. A., Vol.29, No.16, 5181-5186.

[35] L. Lerner. Derivation of the Dirac equation from a relativistic representation of spin, Eur. J. Phys., Vol.17, No.4, 172-175.

[36] T. Kubo, I. Ohba, H. Nitta. A derivation of the Dirac equation in an external field based on the Poisson process, Phys. Lett. A., Vol.286, No.4, 227-230.

[37] H. Cui. arXiv: quant-ph/0102114. 15 Aug 2001, 4 p.

[38] Y. Ng, H. van Dam, arXiv: hep-th/0211002. 4 Feb 2003, 9 p.
[39] M. Calerier, L. Nottale. A scale relativistic derivation of the Dirac equation, Electromagnetic Phenomena, Vol.3, No.1(9), 70-80.

[40] M. Evans. A generally covariant field equation for gravitation and electromagnetism, Found. Phys. Lett., Vol.16, No.4, 369-377.

[41] M. Evans. Derivation of Dirac's equation from the Evans wave equation, Found. Phys. Lett., Vol.17, No.2, 149-166.

[42] V. Simulik, I. Krivsky. Relationship between the Maxwell and Dirac equations: symmetries, quantization, models of atom, Rep. Math. Phys., Vol.50, No.3, 315-328.

[43] V. Simulik, I. Krivsky. Classical electrodynamical aspect of the Dirac equation, Electromagnetic Phenomena, Vol.3, No.1(9), 103-114.

[44] V. Simulik. in "What is the electron?", edit. V. Simulik., Montreal, Apeiron, 2005.

[45] J. Elliott, P. Dawber. Symmetry in Physics, Vol.1, Macmillian Press, London, 1979.

[46] B. Wybourne. Classical groups for Physicists, John Wiley and sons, New York, 1974.

[47] H. Bondi. Negative mass in general relativity, Rev. Mod. Phys., Vol.29, No.3, 423-428.

[48] E. Recami, G. Zino. About new space-time symmetries in relativity and quantum mechanics, Nuovo Cim. A., Vol.33, No.2, 205-215.

[49] G. Landis. Comments on negative mass propulsion, Journal of Propulsion and Power, Vol.7, No.2, 304.

[50] R. Wayne. Symmetry and the order of events in time, A proposed identity of negative mass with antimatter, Turk. J. Phys., Vol.36, No.2, 165-177.

[51] W. Kuellin, P. Gaal, K. Reimann, T. Worner, T. Elsaesser, R. Hey. Coherent ballistic motion of electrons in a periodic potential, Phys. Rev. Lett., Vol.104, No.14, 146602(1-4).

[52] V. Neznamov. On the theory of interacting fields in the Foldy-Wouthuysen representation, Phys. Part. Nucl., Vol.37, No.1, 86-103.

[53] F. Gaioli, E. Alvarez. Some remarks about intrinsic parity in Ryder's derivation of the Dirac equation, Am. J. Phys., Vol.63, No.2, 177-178. 
[54] S. Efthimiades. arXiv: quant-ph/0607001v3. 15 Jan 2011, 8 p.
[55] G. D'Ariano, P. Perinotti. arXiv: 1306.1934 [quant-ph] 8 Jun 2013, 17 p. 